\title{
Towards a Theory of Analytical Behaviour: A Model of Decision-Making in Visual Analytics
}

\author{
Paul Booth \\ University of Southampton \\ pmb1g11@soton.ac.uk
}

\author{
Nicholas Gibbins \\ University of Southampton \\ nmg@ecs.soton.ac.uk
}

\author{
Spyros Galanis \\ University of Southampton \\ s.galanis@soton.ac.uk
}

\begin{abstract}
This paper introduces a descriptive model of the human-computer processes that lead to decision-making in visual analytics. A survey of nine models from the visual analytics and HCI literature are presented to account for different perspectives such as sense-making, reasoning, and low-level human-computer interactions. The survey examines the people and computers (entities) presented in the models, the divisions of labour between entities (both physical and role-based), the behaviour of both people and machines as constrained by their roles and agency, and finally the elements and processes which define the flow of data both within and between entities. The survey informs the identification of four observations that characterise analytical behaviour - defined as decision-making facilitated by visual analytics: bilateral discourse, divisions of labour, mixed-synchronicity information flows, and bounded behaviour. Based on these principles, a descriptive model is presented as a contribution towards a theory of analytical behaviour. The future intention is to apply prospect theory, a economic model of decision-making under uncertainty, to the study of analytical behaviour. It is our assertion that to apply prospect theory first requires a descriptive model of the processes that facilitate decision-making in visual analytics. We conclude it necessary to measure the perception of risk in future work in order to apply prospect theory to the study of analytical behaviour using our proposed model.
\end{abstract}

\section{Introduction}

The fields of visual analytics and visualisation cover a broad range of topics, providing insight into computational and cognitive processes [1, 2, 3, 4]. The technologies and techniques associated with visual analytics (VA) are used in a wide range of applications within industries such as finance, marketing, business intelligence, education, cyber-security, and healthcare. Such tools address the challenges identified by
Cook and Thomas in their VA research agenda [1] from 2005, to obtain insights that directly support assessment, planning and decision-making. In the time Since the agenda was published, new research has extended the understanding of reasoning, sense-making, task-analysis and also introduced behavioural economic concepts of cognitive bias and framing effects to VA.

Decision-making is a broad topic that spans many fields, but one which has been under-examined and not formally defined in VA research. The real-world application of visual analytics and visualisation techniques to decision-making are commonplace. Stock trading is perhaps the most obvious example; traders interact with visualisations to inform their investment choice resulting in an action to buy or sell - all of which occurs within the same software. Economics researchers have observed, modelled, and measured peoples' decision-making for decades. Some experiments have even examined the application of VA to economic decision-making $[2,5,6]$, finding VA provides effective decision support through increased confidence, learning, exploration and leads to better investment choices. However, decision-making is not yet characterised in VA as it has in economics by descriptive models such as prospect theory. Furthermore, economic models do not account for known representation effects that effect decision-making [7]. Therefore, we identify a gap in the study of decision-making facilitated by VA which we define by the term analytical behaviour.

\subsection{Decision-making}

This paper approaches the study and characterisation of decision-making in visual analytics from an economics perspective. Research into cognitive and perceptual processes have largely focused on perceptual accuracy $[8,9,10]$, guiding the design of tools towards increasing their effectiveness and efficiency. However, although some of the psychological and cognitive biases that lead to the 'irrational' behaviour identified by economists [11] are known to VA researchers, there 
is no equivalent guidance on optimising VA tools for decision-making.

Economics research has shown people to be systematic and therefore predictably-irrational in their decision-making processes using prospect theory[12]. However, prospect theory does not account for the visual representation of data as a factor in the process - nor to our knowledge does any other economic model of behaviour. In 1979, when Kahneman and Tversky first introduced Prospect Theory - a descriptive model of choice under uncertainty - there were far fewer decisions being informed by data visualisations compared to today. The ubiquity of VA tools used in decision-making is reason enough to account for them in a descriptive view of the decision-making process.

A recent study within VA into representation effects examined visualisation as a factor in decision-making by reapplying the same general method and prospects as Kahneman and Tversky. In the original prospect theory experiment [12], participants were given different classes of problems presented as written numbers of risk and return values (i.e. choose between a $50 \%$ chance of gaining $\$ 1000$ or $\$ 500$ with certainty). The more recent VA experiment [7] presented similar risk/return prospects in chart form and used different data, yet the results align closely with the results from the original prospect theory experiments; people are more likely to be risk-averse in the domain of gains and risk-seeking when it comes to losses. The study also provides empirical evidence of a significant interaction between the visual representation of data and the perception of value in analytical behaviour.

In the context of modelling stock market trading, once representation effects can be accounted for, the application of prospect theory still depends on knowing the user's perceived valuation of risk and return. We speculated that inputs for prospect theory's value function could be taken from observations about a user's decision (e.g. the price of a trade) and from information about the interactions that preceded their final choices such as the graphical display and the underlying data being presented. For the risk value in prospect theory's weighting function, we anticipated a difference between objectively calculating risk and how risk would perceived when implied in a line chart [7].

In other words, we suggest that to apply prospect theory to decision-making scenarios in VA it is necessary to observe both decision values and a the user's task in a semantically meaningful way (i.e. buy or sell), capture the underlying data being presented and also account for the effect of visualisations on a person's perception of value. It is also necessary to make assumptions about the cognitive and computational processes that lead up to a person's final decision, given that these cannot be observed in the same way as decision values or actions. This paper contributes a descriptive model of behaviour which to be empirically tested in future work, as a step towards a theory of analytical behaviour. Measuring and predicting risk perception is outside of the scope.

\section{Survey}

The following section presents a survey of nine models from across the VA and HCI literature relating to analytical reasoning, sense making, and human-computer interaction processes. The VA models were identified from a review of more than forty visual analytics papers specifically referring to analytical reasoning or sense-making. The HCI models are based on a review of more than thirty HCI papers referring to fundamental models and frameworks of interaction processes. None of the models specifically relate to decision-making but they each offer a view into the entities, actions, and processes that facilitate the type of decision-making scenarios in VA we are interested in.

From an initial analysis of all models, we are able to identify four common components that are relevant to decision-making in all the models of the survey; the entities present in the model (i.e. person, computer), divisions of labour between entities, their behaviour (meaning their actions as defined by their role and agency), plus the elements and processes which define the flow of data and information both within and between entities.

The survey summary presented in Table 1 shows five models from the VA literature and four from Human-Computer Interaction (HCI). The HCI models serve to illustrate (and to varying degrees somewhat describe) the low-level interactions that exist - but are not shown - in the normative VA models. The summary table provides the publication reference for each model and the model title. A model type states if it is normative (i.e. what users should do), descriptive (it more closely predicts what people actually do), or mixed. Field indicates whether the model is from VA or HCI, entities observes whether human or computer entities are explicitly presented in the model. A count of explicit divisions of labour is given, along with a coding of the type of information flow that is either unidirectional in the case of the HCI models, iterative in the case of sense-making, or containing linear or mixed-initiative elements. Finally, a summary of the main elements and processes are provided - these are simply the key titles and annotations that appear within the models. 


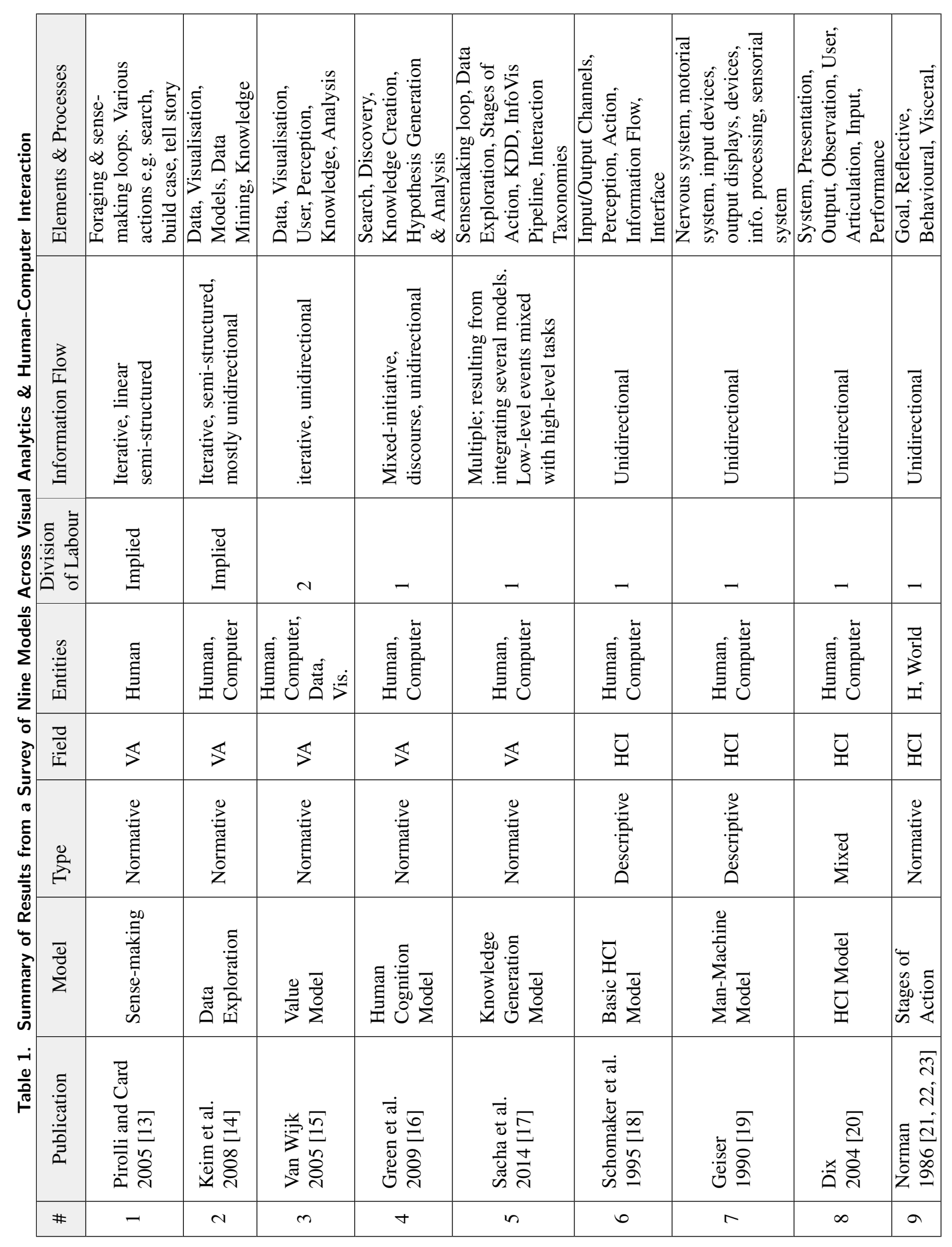




\subsection{Observations from the Survey}

We find that all of the models from the visual analytics literature provide a normative view of reasoning processes; they show what users should $d o$ and not what they actually do. For the sense-making model, Pirolli and Card [13] set their model apart from other reports that 'provide normative or prescriptive views on intelligence analysis' by basing the model on an empirical study of analysts' working processes. However, their conceptual model represents 'an approximate data flow observed across several analysts' [24], offering a more informed - but ultimately still a normative view - of behaviour relating to the collection and organization of information, some of which may precede analytical behaviour.

From the visual analytics models, only two explicitly show two entities. Both the Human Cognition Model [25] and the Knowledge Generation Model [17] show clearly defined human and computer entities but in different ways. The former presents several patterns of discourse between the two entities, whereas the latter groups several different models under human or computer entities without fully integrating them. All the HCI models present the same two entities (human and computer), showing physical features and implied constraints of the entities as well as the discourse they are engaged in.

There are no divisions of labour or mediating objects (e.g. screen, mouse, keyboard) explicitly illustrated in the majority of the VA models. In contrast there does exist an explicitly rendered division of labour between agents in all the HCI models. However, only Dix's model [20] puts a physical space between entities that is mediated by artefacts.

The behaviour observed in the surveyed VA models includes include searching, evaluating, presenting, data mining, and the production of knowledge artefacts. Differences in the behaviour illustrated between models stems from differences in HCI and VA perspectives, and also from the subject under observation. Pirolli and Card's sense-making diagram [13] is based on a study of intelligence analysts, while the HCI models describe low-level interactions that form the basis for high-level tasks and behaviours shown in VA models.

There are some common elements and processing concepts across models, while others are unique to a single model. In all of the HCI models but only one of the visual analytics models (from Green and Sacha [16]), the physical structure constrains the flow of information, rendering it unidirectional. This illustrates the true way that information, during a human-computer analytic discourse, moves in one direction; from visualisations on screen to the human eye and mind, then from the mind into physical interactions - an input the computer then responds to by changing what is displayed on screen.

\subsection{Interaction Taxonomies}

As part of the survey we also reviewed literature classifying low-level interactions and high-level tasks, which relate to the behaviour either implied or illustrated in most of the models in the survey.

Task analysis in VA has covered a broad range of topics and challenges, including; task taxonomies [26, 27, 28], interaction $\log$ analysis [29], identifying information visualisation activities [30], classifying activity patterns [31], creating a typology of abstract visualisation tasks [32], and characterising the tasks undertaken by intelligence analysts [33]. Researchers have also used 'provenance trail' vocabularies in collaborative analysis [34], as an aid in teaching [35], to provide visualisation recommendations [36], and to improve the contextual awareness of a VA system [37].

A continuing challenge in task research is activity recognition; mapping low-level events to high-level goals. The problem stems from the fact that goals and tasks are more likely to be domain-specific whereas lower-level events and actions are domain-agnostic. When a person is engaged in a reasoning or sense-making process, their tasks are more exploratory and therefore not as easily inferred. Comparatively, actions relating to decision-making are easier to observe when they relate to simple actions in an interface such as buying, selling, or bidding. Other non-economic actions could also be detected, such as changes to physical behaviour immediately after reviewing health data from a fitness-tracker application for instance. Our assertion is that decision-making within the scope of the study of analytical behaviour can be observed and descriptively modelled - unlike the goals relating to normative tasks that need to be generalised from observed actions, such as sense-making and reasoning.

\section{Towards a Theory of Analytical Behaviour: Observations}

The following section presents a set of observations proposed as the initial principles of analytical behaviour, developed from our survey of VA and HCI models in 1. The principles describe the entities, structure and processes in VA that facilitate decision-making. Further empirical evidence is required to support that the proposed principles are all necessary and sufficient to describe analytical behaviour. Our present intention is therefore only to propose a set of principles to subject 
to further experimentation and evaluation towards the goal of creating a theory of analytical behaviour. The proposed principles are: bilateral discourse, divisions of labour, mixed-synchronicity information flows, and bounded behaviour. An overview of each principle is given below, followed by a visual model to illustrate most of the principles at work and as a tool for the design and evaluation of analytical behaviour.

- bilateral discourse defines there to be two entities engaged in an analytic discourse; one human user and one computer system. Additional devices mediate communication between the two.

- divisions of labour outlines the physical space between human and machine, but also separations in the work they are ideally suited to - creative thinking and cognition are the strengths of people, but computers are far superior when it comes to memory accuracy and capacity or computations at speed and scale.

- mixed-synchronicity data flows describe the fundamental dynamics of how information (and data) flow between the two entities and across the division of labour. The majority of models in the survey present a unidirectional flow of information; from a computer screen, across a physical division to a user's eye, and brain where cognitive processes form decisions that are turned into actions as an input via a mouse or keyboard that goes back to the computer. The principle of mixed-synchronicity information flow states that there are two types of unidirectional information flow; synchronous (which blocks other information flows) and asynchronous (which allows other information to flow through while the original process finishes).

- bounded behaviour highlights that each entity is limited in its own capacity to process information and act. The principle draws on the concept of bounded rationality from economics [38, 39]. Bounded behaviour is inclusive of other biases [40] and framing effects also [41, 42, 43]

\subsection{Bilateral Discourse}

In two of the visual analytics models and all of the HCI models surveyed in Section 2, entities are seen to explicitly engage in a discourse with one another. Cook and Thomas refer to analytic discourse as "the technology-mediated dialogue between an analyst and his or her information to produce a judgement about an issue" [1, p.38]. Adding that it is an "iterative and evolutionary process by which a path is built from definition of the issue to the assembly of evidence and assumptions to the articulation of judgements". Both 'bilateral' and 'analytic' discourse underscore the relationship between the user and the computer is collaborative and dynamic as a function. However, analytic discourse infers sense-making whereas bilateral discourse is intended to describe a more fundamental structural dynamic in all processes that lead to analytical behaviour.

The two entities engaged in a discourse are separated physically by a barrier of communication mediated by physical input/output devices. Bilateral in this context is taken to mean a system of two sides - man and machine as two halves of something that is more than the sum of its parts. The two entities are bridged by peripherals like display monitors that act as impartial translators between entities. There are some instances where decisions made by more than two entities, but these situations are outside of the present scope. Our focus is on a unit of analysis confined to one user and one computer (interface) and any artefacts that mediate their interaction.

Specifically, bilateral defines a process that relates to two sides of something, in this case the discourse between the user and the computer. The term Bilateral should not be taken to imply additional constraints on the role each entity plays, their level of intelligence, or the agency of either entity at any point in the process. Both entities are free to change their role to support the goal of optimising decision-making, the discourse still remains bilateral. Either entity can act as the initiator of a task or process - there is no boundary to the level of autonomy or role that either entity can take. The computer could either be passive or active with varying degrees of intelligence $[44,45]$. Either entity can initiate a new flow of information depending on the autonomy and role of the entity, providing the flexibility to describe different discourse patterns such as those seen in the Human Cognition Model [16].

\subsection{Divisions of Labour}

Conceptually, a division of labour describes the point of separation between entities. It also determines the boundary of an entity's functional capacity within an economic system. The concept of divisions of labour in economics can be traced back to Adam Smith's commentary on the shared working practises observed in a pin factory in the late 18th-century $[46,47]$. The topic of labour division has already permeated the visual analytics literature when it was used to explain how visual analytics "integrates scientific disciplines to 
improve the division of labour" (between humans and computers) [14, p.157].

In financial VA tools, divisions of labour depend on the type of user - professionals may need more specific computational assistance and more control and detailed market analysis while consumers with less knowledge and less time to spend want simple insights and clear guidance. Consumer-focused investment mobile apps try to reduce the complexity of data visualisations and simplify the decision-making process by providing more intuitive portfolio projections and uncomplicated investment options.

Of the surveyed models in Table 1, not all of them show a single division of labour. The Value Model [14] is the only model to include two divisions; one between Data and Visualisation, the other between Visualisation and the User. This could be interpreted from Cook and Thomas' description of analytic discourse being a "technology-mediated dialogue between an analyst and his or her information". However, while Cooks and Thomas' definition and Keim's value model agree, their view is at odds with the single division of labour presented by the HCI and two other visual analytics models that group data and visualisation within the computer entity. Comparatively, the Human Cognition Model (HCM) [25] and the Knowledge Generation Model (KGM) [17] both explicitly show a single division of labour.

The role of some recent analytics systems have introduced the capability to recommend graphs based on the tasks a user is performing [48, 49, 44]. Other VA research is evolving the previous limits of a computers role, and changing the human role, with developments in mixed-initiative systems $[44,50]$.

\subsection{Bounded Behaviour}

The principle of bounded behaviour asserts that in the process of bilateral discourse, both entities are subject to the physical, computational, informational, psychological, and role constraints that apply to either people or machines. Economics assigns the term bounded rationality to "rational choice that takes into account the cognitive limitations of the decision maker" [38, p.291]. A central theme in behavioural economics, bounded rationality highlights the limitations of the decision-maker in the process of decision-making [51, 52, 38, 39]. The principle of bounded behaviour introduced in this section borrows extensively from the economic concept of bounded rationality.

Of all of the visual analytics models surveyed in Table 1, entities are always subject to constraints that restrict behaviour. For example, there is a finite amount of information that can be encoded into a chart before it either becomes too difficult for the eyes and mind to process or it becomes too big to show on a screen. Visual complexity is one of several known challenges with visual perception $[10,53,54]$.

The constraints of bounded behaviour also relate to well documented biases in economics such as availability bias, anchoring, confirmation bias and recency bias $[55,56,40]$. Research in VA has already begun to identify and compensate for selection bias [57], but the current effort to combat bias is vastly outweighed by the number biases known to economic researchers.

Behaviour could also be bound by representation effects. Recent experimental evidence specifically measuring the affect of visualisations on analytical behaviour suggests that changes to a graph's proportion and the use of a dual-line chart (compared to two single line-charts) affects the choices of a significant number of people [7]. This example goes beyond the perceptual limitations previously mentioned, which are only concerned with the perceptual accuracy and not how it affects decision-making. When considering a choice under uncertainty, research in economics suggests that people go through a process of framing and evaluating values and risk, such that most people are more sensitive to losses than gains [58, 59, 41].

As an example of bounded behaviour in the real-world, a user after viewing the historical and current prices of a listed stock will make a choice to buy or sell (or do nothing). The user cannot possibly be aware of all qualitative or quantitative data relating to market trends - she is bound by physical limitations. Moreover, the presentation method itself may create a representation effect that leads the user to inaccurately interpret the level of risk or return, making one choice potentially more appealing than another. Also, the user's reference point could be influenced by framing effects [59, 60], leading to changes in how gains and losses are perceived that alter a person's final choice. Taken together there are many ways a user's behaviour can be bound by the limitations of perception, psychology and cognition.

\subsection{Mixed-Synchronicity Information Flows}

The fourth principle of mixed-synchronicity information flows describes two types of information "fluidity"; synchronous and asynchronous. Synchronous information processing blocks other information-flows until processing has completed; therefore stopping one or more entities from completing or initiating a task. Asynchronous information by comparison does not block the processing or performance of tasks. To our knowledge, no studies have yet examined the 
'synchronicity' of human responses in relation to the accuracy of value judgements or decision-making in the context of VA. However, there are plenty of everyday examples of mixed-synchronicity easily observed, such as reading sheet music while playing an instrument, or driving a car while talking to passengers.

Regardless of processing capacity, the flow of information is unidirectional at the lowest level of interaction. In other words, information can only flow in one direction between the user and the computer; from computer screen to the users visual perception and their mind. Then, utilising peripherals, the user interacts with the computer which responds and reflects the change on-screen. The HCI models in the survey demonstrate this at a low-level not seen in the VA models. The high-level tasks in sense-making and reasoning are still made up of a series of the same lower-level interactions that are unidirectional and either synchronous or asynchronous.

Depending on who or what is processing information (or data), the type and rate of occurrences of are likely to differ. For instance, when a user moves an input device (e.g. a mouse) the computer is not blocked from doing other processing. However, a person is not able to write at all well while simultaneously trying to also listen to (and process) unrelated audio information. Both example processes are synchronous - the two tasks cannot occur at the same time, one blocks the other. Comparatively but computationally, a high number of SVG (Scalable Vector Graphics) elements rendered to a web page can cause the web browser to "hang" and therefore temporarily block the user from interacting until processing is complete. It is possible therefore, for a person or computer to 'block' both themselves and one another from processing or initiating an information flow.

\section{Analytical Behaviour Model}

In this section we present a model of analytical behaviour based on the principles introduced in the previous section. The Analytical Behaviour (AB) Model features two entities with a physical divide between them. Two peripheral devices mediate the communication divide between entities (e.g. input/output devices $\mathrm{Y}$ and $\mathrm{X}$ in Figure 1. These are assumed to be a display monitor (X) and a standard mouse and keyboard (Y), but could be replaced with other hardware such as touch screen on a mobile device, a voice interface or specialist VR/AR equipment. In the case of a mobile device, the touch screen interface, computer, and screen are all the same physical device. The model in Figure 1 is still valid, the flexibility

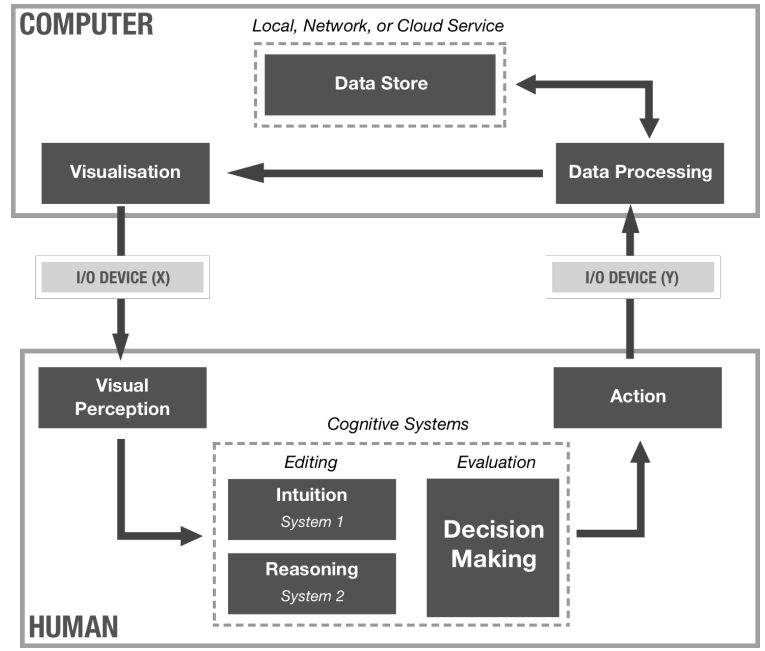

Figure 1. The Analytical Behaviour Model complies with the four principles of Analytical Behaviour and also integrates dual-systems theory while drawing structural influence from $\mathrm{HCl}$ models.

remains also for the two peripherals to be combined into one element to represent a shared screen and interface. Regardless, the flow of information would still be unidirectional and the principles still valid.

The flow of information is unidirectional except for the data store in the AB Model. However, data process and the data store could just as well have been a combined component. Either way, unidirectional data flow is not a principle itself but an observation of the flow driven by the input/output devices. Information flows from the monitor to the user, and from the user's mouse or keyboard to the computer - it cannot flow in reverse. The principle of mixed-synchronicity does apply to the data store and data processing flow, the realities of which are entirely dependant on the architecture of an application. Therefore, the data store and flow shown in the AB Model are one of several possible abstractions.

The dual-systems theory of cognition is a concept from economics [61] that was in the work on prospect theory [39]. Dual-process theory defines two modes of cognition; intuition and reasoning. The theory has been used not just in economics but in cognitive and social psychology, so widely has it been applied that providing a coherent view of the theory is challenging [62]. The shared view however, is that there are two systems of thinking labelled as System 1 and System 2 that align to intuition and reasoning respectively.

Using dual-systems theory in the $\mathrm{AB}$ model is a logical step towards a theory of analytical behaviour and the application of prospect theory to decision-making in VA. Intuition and reasoning in dual-systems theory are 
two ways people solve problems; Kahneman suggests that intuition most resembles perception in that they are both subject to reference dependence and both "come to mind spontaneously" [39]. Reasoning is a slower, more conscious process by comparison. In the VA models from our earlier survey, "knowledge" and "insight" are often used as a way to generalise about cognitive processes. Similarly, HCI models often generalise cognitive processes as 'cognition', which is much broader than the VA models. Dual-systems theory offers a more complete view of cognition that aligns to prospect theory while not entirely displacing existing notions of cognition from VA or HCI.

\section{The Analytical Behaviour Model as a Web Framework}

To explore the practical use of the AB model, a small JavaScript library was created to observe, describe, and potentially even predict analytical behaviour in a web browser. The library is based entirely on the AB Model and design to observe and store information about the SVG visualisations used on screen, their underlying data, and the user's action (to buy or sell stocks in a single page web-app). A simulation was created based on a previous experiment into representation effects involving three-hundred participants [7] where participants made a series of choices between different pairs of stock investment opportunities presented on the screen as two line graphs. The premise being that the user is selecting a stock to invest, under uncertainty, given the limited information about each stock's price over the last thirty days and their perceived risk profiles.

The completed web framework provides some validation of the $\mathrm{AB}$ Model as a descriptive model of behaviour by capturing SVG information, the underlying data being visualised on-screen, as well observing the user's button clicks. The cognitive systems of a user is not directly observable, which is where prospect theory applies as model of choice under uncertainty.

However, when we tried to apply the value and weighting functions of prospect theory to analytical behaviour using the framework, a unmeasured perceptual issue became evident. Even though we can account for some representation effects, the risk of a stock - when visualised in a line chart - is implied by the user from the shape of the line. In the original prospect theory experiments by Kahneman and Tversky, these were explicit written probabilities. Therefore, to apply prospect theory using the AB framework the user's perception of risk must be accounted for and converted into a value for use in the prospect theory formula.
We know from a previous experiment [7] that the height-to-width ratio of a graph effects the decisions of users. Representation effects explain the variance between users' choices when they view different styles of the same data, but they do explain the precise risk value that the user actually perceives.

\subsection{Summary}

This paper presented a model and characterisation of analytical behaviour, defined as decision-making facilitated by visual analytics techniques and technologies [7]. In an analysis of VA models in the survey we focused on four elements; the entities present in the model (i.e. human analyst or computer), divisions of labour, behaviour (roles, agency), and the elements and processes that define the flow of data in the models.

The result of the survey analysis is a set of observations put forward as principles; bilateral discourse, divisions of labour, bounded behaviour, and mixed-synchronicity data flows. These principles would benefit from additional supporting evidence in future, in the form of empirical evidence.

From these principles, a model of analytical behaviour is developed. Whether explicitly shown in a model or not - two entities are always present in the analytic discourse, even if they are implied as is the case with the normative VA models of reasoning and sense-making. A physical divide exists between the user and the computer which is represented explicitly in all the HCI models but only in two of the VA models. The majority of the models surveyed also indicate a unidirectional flow of information, which is not itself a core principle.

The visual analytics models we surveyed tend to describe the user's behaviour normatively, in terms of tasks, goals, or generalised actions. HCI models, by contrast, show low-level interactions that more closely describe the fundamental mechanics underlying human-computer discourse implied in all VA processes reasoning, sense-making, and also analytical behaviour.

We have not been able to illustrate all four principles in the model. Mixed-synchronicity information flows can only be shown by animating the model or with alternative layouts. The four principles presented in this chapter align closely to the low-level structure seen in HCI models which, like the AB Model presented in Figure 1, illustrate a unidirectional flow of information and a single division of labour between two entities. We have discussed mixed-synchronicity information flows in detail and how the behaviour of human and (intelligent) machines can experience different 
types bounded behaviour due to their unique physical, computational or psychological limitations. We have aligned the concept of behavioural constraints (in the principle of bounded behaviour) to the economic theory of 'bounded rationality', providing examples to support our assertions of this concept in the context of analytical behaviour.

To complete our descriptive model of analytical behaviour with the application of prospect theory, future work must focus on understanding users' perception of risk in line charts. One of the required parameters in the value and weighting functions of prospect theory are explicit risk values [12]. However, risk is only implied in line charts. Before prospect theory can be applied it is necessary to measure and predict the perception of risk from a line chart, which to our knowledge has not been addressed in either the economic or VA literature.

\section{References}

[1] K. Cook and J. Thomas, "Illuminating the Path: The Research and Development Agenda for Visual Analytics," tech. rep., 2005.

[2] A. Savikhin, R. Maciejewski, and D. S. Ebert, "Applied Visual Analytics for Economic Decision-Making," IEEE Symposium on Visual Analytics Science and Technology, pp. 107-114, 2008.

[3] T. Munzner, "Visualization," in Fundamentals of Computer Graphics, pp. 675-707, CRC Press, July 2009.

[4] G.-D. Sun, Y.-C. Wu, R.-H. Liang, and S. X. Liu, "A Survey of Visual Analytics Techniques and Applications: State-of-the-Art Research and Future Challenges," Journal of Computer Science and Technology, vol. 28, no. 5, pp. 852-867, 2013.

[5] S. Rudolph, A. Savikhin, and D. S. Ebert, "FinVis: Applied visual analytics for personal financial planning," IEEE Symposium on Visual Analytics Science and Technology, VAST 2009, pp. 195-202, 2009.

[6] A. Savikhin, H. C. Lam, B. Fisher, and D. S. Ebert, “An Experimental Study of Financial Portfolio Selection with Visual Analytics for Decision Support," 44th Hawaii International Conference on System Sciences (HICSS), 2011, pp. 1-10, 2011.

[7] P. Booth, N. Gibbins, and S. Galanis, "Representation Effects and Loss Aversion in Analytical Behaviour: An Experimental Study into Decision Making Facilitated by Visual Analytics," in The Hawaii International Conference on System Sciences, Hawaii International Conference on System Sciences, Jan. 2018.

[8] W. S. Cleveland and R. McGill, "Graphical Perception: Theory, Experimentation, and Application to the Development of Graphical Methods," Journal of the American Statistical Association, pp. 531-554, 1984.

[9] C. Ware, Information Visualization. Perception for Design, Morgan Kaufmann, 2004.

[10] J. Talbot, V. Setlur, and A. Anand, "Four Experiments on the Perception of Bar Charts," IEEE Transactions on Visualization and Computer Graphics, vol. 20, no. 12, pp. 2152-2160, 2014.
[11] D. Ariely, Predictably Irrational: The Hidden Forces that Shape Our Decisions. HarperCollins UK, Mar. 2009.

[12] D. Kahneman and A. Tversky, "Prospect Theory: An Analysis of Decision Under Risk," Econometrica: Journal of the Econometric Society, pp. 263-291, 1979.

[13] P. Pirolli and S. Card, "The Sensemaking Process and Leverage Points for Analyst Technology as Identified Through Cognitive Task Analysis," in Proceedings of international conference on intelligence analysis, pp. 2-4, 2005.

[14] D. Keim, G. Andrienko, J. Fekete, C. Görg, J. Kohlhammer, and G. Melançon, "Visual Analytics: Definition, Process, and Challenges," Information Visualization, pp. 154-175, 2008.

[15] J. J. van Wijk, "The Value of Visualization," in IEEE Visualization, pp. 79-86, IEEE, 2005.

[16] T. M. Green, W. Ribarsky, and B. Fisher, "Building and Applying a Human Cognition Model for Visual Analytics," Information Visualization, vol. 8, no. 1, pp. 1-13, 2009.

[17] D. Sacha, A. Stoffel, F. Stoffel, B. Kwon, G. Ellis, and D. Keim, "Knowledge Generation Model for Visual Analytics," IEEE Transactions on Visualization and Computer Graphics, vol. 20, no. 12, pp. 1604-1613, 2014.

[18] L. Schomaker, J. Nijtmans, A. Camurri, F. Lavagetto, P. Morasso, and C. Benoit, "A Taxonomy of Multimodal Interaction in the Human Information Processing System," tech. rep., 1995.

[19] G. Geiser, Mensch-Maschine-Kommunikation. München : R. Oldenbourg, 1990

[20] A. Dix, Human-Computer Interaction. Pearson Education, 3rd ed., 2004.

[21] D. Norman, "Cognitive Engineering," in User Centered System Design (D. Norman and S. Draper, eds.), London: Lawrence Erlbaum Associates, 1986.

[22] D. A. Norman, "Design principles for cognitive artifacts," Research in Engineering Design, vol. 4, pp. 43-50, Mar. 1992.

[23] D. Norman, The Design of Everyday Things. Broadway Business, 1988.

[24] P. Pirolli and D. Russell, "Introduction to this Special Issue on Sensemaking," Human-Computer Interaction, vol. 26, no. 1, pp. 1-8, 2011.

[25] T. M. Green and W. Ribarsky, "Using a human cognition model in the creation of collaborative knowledge visualizations," Defense and Security 2008: Special Sessions on Food Safety, Visual Analytics, Resource Restricted Embedded and Sensor Networks, and 3D Imaging and Display, vol. 6983, p. 69830C, Apr. 2008.

[26] B. Shneiderman, "The Eyes Have It: A Task by Data Type Taxonomy for Information Visualizations," IEEE Symposium on Visual Languages, pp. 336-343, 1996.

[27] B. Lee, C. Plaisant, C. Parr, J. Fekete, and N. Henry, "Task taxonomy for graph visualization," in Proceedings of the 2006 AVI workshop on BEyond time and errors: novel evaluation methods for information visualization, (New York), pp. 1-5, ACM, 2006.

[28] J. Heer and B. Shneiderman, "Interactive Dynamics for Visual Analysis," Queue, vol. 10, no. 2, 2012. 
[29] H. Guo, S. Gomez, C. Ziemkiewicz, and D. Laidlaw, "A Case Study Using Visualization Interaction Logs and Insight Metrics to Understand How Analysts Arrive at Insights," IEEE Transactions on Visualization and Computer Graphics, vol. 22, no. 1, pp. 51-60, 2016.

[30] R. Amar, J. Eagan, and J. Stasko, "Low-level Components of Analytic Activity in Information Visualization," IEEE Symposium on Information Visualization, pp. 111-117, 2005.

[31] D. Gotz and M. Zhou, "An Empirical Study of User Interaction Behavior During Visual Analysis," Tech. Rep. RC24525, 2008.

[32] M. Brehmer and T. Munzner, "A Multi-Level Typology of Abstract Visualization Tasks," IEEE Transactions on Visualization and Computer Graphics, vol. 19, no. 12, pp. 2376-2385, 2013.

[33] Y. Kang and J. Stasko, "Characterizing the Intelligence Analysis Process," IEEE Conference on Visual Analytics Science and Technology, pp. 21-30, 2011.

[34] J. Lu, Z. Wen, S. Pan, and J. Lai, "Analytic Trails: Supporting Provenance, Collaboration, and Reuse for Visual Data Analysis by Business Users," in IFIP Conference on Human-Computer Interaction, pp. 256-273, Springer Berlin Heidelberg, Springer Berlin Heidelberg, 2011.

[35] C. Silva, E. Anderson, E. Santos, and J. Freire, "Using VisTrails and Provenance for Teaching Scientific Visualization," Computer Graphics Forum, vol. 30, pp. 75-84, Mar. 2011.

[36] D. Gotz and M. Zhou, "Characterizing Users' Visual Analytic Activity for Insight Provenance," Information Visualization, vol. 8, no. 1, pp. 42-55, 2009.

[37] M. Derthick and S. Roth, "Enhancing Data Exploration with a Branching History of User Operations," Knowledge-Based Systems, vol. 14, no. 1, pp. 65-74, 2001.

[38] H. Simon, Models of Bounded Rationality: Empirically Grounded Economic Reason. MIT Press, 1982.

[39] D. Kahneman, "Maps of Bounded Rationality: Psychology for Behavioral Economics," The American Economic Review, vol. 93, no. 5, pp. 1449-1475, 2003.

[40] T. Gilovich, D. Griffin, and D. Kahneman, Heuristics and Biases. The Psychology of Intuitive Judgment, Cambridge University Press, 2002.

[41] I. Levin, S. Schneider, and G. Gaeth, "All Frames are not Created Equal: A Typology and Critical Analysis of Framing Effects," Organizational Behavior and Human Decision Processes, vol. 76, no. 2, pp. 149-188, 1998.

[42] J. Hullman and N. Diakopoulos, "Visualization Rhetoric: Framing Effects in Narrative Visualization," IEEE Transactions on Visualization and Computer Graphics, vol. 17, no. 12, pp. 2231-2240, 2011.

[43] D. J. Koehler and N. Harvey, Blackwell Handbook of Judgment and Decision Making. Oxford: Blackwell Publishing, 2004.

[44] K. Cook, N. Cramer, D. Israel, M. Wolverton, J. Bruce, R. Burtner, and A. Endert, "Mixed-Initiative Visual Analytics Using Task-Driven Recommendations," in IEEE Conference on Visual Analytics Science and Technology (VAST), pp. 9-16, IEEE, 2015.

[45] E. Horvitz, "Principles of Mixed-Initiative User Interfaces," in Proceedings of the SIGCHI Conference on Human Factors in Computing Systems, pp. 159-166, ACM, 1999.
[46] A. Smith, An Inquiry Into the Nature and Causes of the Wealth of Nations. MetaLibri, 2007.

[47] P. Samuelson and W. Nordhaus, Economics. New York: McGraw-Hill/Irwin, 19th ed., 2010.

[48] D. Gotz, Z. When, J. Lu, P. Kissa, N. Cao, W. H. Qian, S. X. Liu, and M. Zhou, "HARVEST: An Intelligent Visual Analytic Tool for the Masses," in Proceedings of the First international Workshop on Intelligent Visual Interfaces for Text Analysis, ACM Press, 2010.

[49] M. Voigt, S. Pietschmann, and L. Grammel, "Context-Aware Recommendation of Visualization Components," in Proceedings of the 4th International Conference on Information, Process, and Knowledge Management, pp. 101-109, 2012.

[50] S. Makonin, D. McVeigh, W. Stuerzlinger, K. Tran, and F. Popowich, "Mixed-Initiative for Big Data: The Intersection of Human + Visual Analytics + Prediction," in The Hawaii International Conference on System Sciences, pp. 1427-1436, IEEE Computer Society, Jan. 2016.

[51] H. Simon, "A Behavioral Model of Rational Choice," The Quarterly Journal of Economics, vol. 69, no. 1, pp. 99-118, 1955.

[52] H. Simon, "Theories of Bounded Rationality," Decision and Organization, vol. 1, no. 1, pp. 161-176, 1972.

[53] M. Correll and M. Gleicher, "Error Bars Considered Harmful: Exploring Alternate Encodings for Mean and Error," IEEE Transactions on Visualization and Computer Graphics, vol. 20, pp. 2142-2151, Dec. 2014.

[54] C. Gramazio, K. Schloss, and D. Laidlaw, "The Relation Between Visualization Size, Grouping, and User Performance," IEEE Transactions on Visualization and Computer Graphics, vol. 20, no. 12, pp. 1953-1962, 2014.

[55] A. Tversky and D. Kahneman, "Judgment Under Uncertainty: Heuristics and Biases," Science, vol. 185, no. 4157, pp. 1124-1131, 1974.

[56] R. Nickerson, "Confirmation Bias," Review of General Psychology, vol. 2, no. 2, pp. 175-220, 1998.

[57] D. Gotz, S. Sun, and N. Cao, "Adaptive Contextualization: Combating Bias During High-Dimensional Visualization and Data Selection," Proceedings of the 21st International Conference on Intelligent User Interfaces, pp. 85-95, Mar. 2016.

[58] A. Tversky and D. Kahneman, "The Framing of Decisions and the Psychology of Choice," Science, vol. 211, no. 4481, pp. 453-458, 1981.

[59] A. Tversky and D. Kahneman, "Rational Choice and the Framing of Decisions," Journal of Business, vol. 59, no. 4, pp. S251-S278, 1986.

[60] I. P. Levin, M. A. Snyder, and D. P. Chapman, "The Interaction of Experiential and Situational Factors and Gender in a Simulated Risky Decision-Making Task," dx.doi.org, vol. 122, pp. 173-181, July 2010.

[61] K. E. Stanovich and R. F. West, "Individual Differences in Reasoning: Implications for the Rationality Debate?," Behavioral and Brain Sciences, vol. 23, pp. 645-726, 2000.

[62] J. Evans, "Dual-Processing Accounts of Reasoning, Judgment, and Social Cognition," Annual Review of Psychology, vol. 59, no. 1, pp. 255-278, 2008. 